\title{
SLANTED SYMPLECTIC QUADRANGLES
}

\begin{abstract}
By 'slanting' symplectic quadrangles $\mathrm{W}(F)$ over fields $F$, we obtain very simple examples of non-classical generalized quadrangles. We determine the collineation groups of these slanted quadrangles and their groups of projectivities. No slanted quadrangle is a topological quadrangle.
\end{abstract}

A simple modification of symplectic quadrangles yields examples of nonclassical generalized quadrangles. The finite quadrangles of this type are well known and due to Ahrens-Szekeres and M. Hall, see Payne [15], [16], Payne and Thas $[18,3.1 .3,3.1 .4,3.1 .5,3.2 .6]$; cf. also [1], [2], [4], [5], [19].

We prove that the collineation groups of these 'slanted' quadrangles are inherited from the corresponding symplectic quadrangles (2.4), with the exception of the two smallest thick cases. Furthermore, we compute the groups of projectivities of slanted quadrangles $(3.3,3.4)$; it turns out that all even finitary permutations are projectivities. As a consequence, the slanted quadrangles cannot be turned into topological quadrangles (3.12).

We would like to thank Hendrik Van Maldeghem for requiring a correction in (2.2).

\section{Construction}

Let $F$ be a field, and denote by $P$ and $\mathscr{L}$ the point set and the line set, respectively, of the projective 3 -space $\mathrm{PG}_{3} F$. For $p \in P$, we denote by $\mathscr{L}_{p}=\{L \in \mathscr{L} \mid p \in L\}$ the line pencil through $p$. Let $\pi$ be the (essentially unique) symplectic polarity of $\mathrm{PG}_{3} F$, and let

$$
\mathscr{L}_{\pi}=\left\{L \in \mathscr{L} \mid L^{\pi}=L\right\}
$$

be the set of all absolute lines. Then $\mathrm{W}(F)=\left(P, \mathscr{L}_{\pi}, \epsilon\right)$ is the symplectic quadrangle over $F$ (here we consider lines as sets of points).

The point set $p^{\pi}$ is the star of $p$, i.e. the set of all points joined to $p$ in $\mathrm{W}(F)$.

(1.1) DEFINITION. Choose $s \in P$, and define the slanted symplectic quadrangle $\mathrm{W}(F)^{\langle s\rangle}=\left(P^{\langle s\rangle}, \mathscr{L}^{\langle s\rangle}, \epsilon\right)$ by

$$
P^{\langle s\rangle}=P \backslash s^{\pi} \quad \text { and } \quad \mathscr{L}^{\langle s\rangle}=\left(\mathscr{L}_{\pi} \backslash \mathscr{L}_{\mathrm{s}}\right) \cup\left(\mathscr{L}_{\mathrm{s}} \backslash \mathscr{L}_{\pi}\right) .
$$

Thus the new point set $P^{\langle s\rangle}$ is just an affine 3-space $\mathrm{AG}_{3} F$, and the line set 
$\mathscr{L}^{\langle s\rangle}$ consists of the absolute lines not contained in the hyperplane $s^{\pi}$ and of one parallel class $\mathscr{L}_{s} \backslash \mathscr{L}_{n}$ of $\mathrm{AG}_{3} F$; we refer to the lines in this parallel class as special lines.

Since the projective symplectic group $\mathrm{PSp}_{4} F$ is transitive on $P$, the isomorphism type of the incidence structure $\mathrm{W}(F)^{\langle s\rangle}$ does not depend on $s$, and occasionally we write just $\mathrm{W}(F)^{\diamond}, P^{\diamond}$ and $\mathscr{L}^{\diamond}$. For finite fields $F$, the following result, or rather an equivalent statement for a different model of $\mathrm{W}(F)^{\langle s\rangle}$, was proved by Ahrens-Szekeres, $\mathrm{M}$. Hall and others, see Payne and Thas $[18,3.1 .5]$, Payne $[15, \S 5]$.

In fact, using the concept of regular points, one could also 'slant' some other (finite) quadrangles apart from those of the symplectic type (cf. Payne and Thas $[18,1.3])$. But this will not be pursued here.

(1.2) LEMMA. For every field $F$, the incidence structure $\mathrm{W}(F)^{\langle s\rangle}$ is a generalized quadrangle. It is thick if and only if $|F|>2$.

Proof. Clearly, any two distinct points are joined by at most one line. Since $\mathrm{W}(F)$ contains no triangle, any triangle in $\mathrm{W}(F)^{\langle s\rangle}$ has at least one special line $S \in \mathscr{L}_{s} \backslash \mathscr{L}_{\pi}$. As distinct special lines are disjoint in $\mathrm{W}(F)^{\langle s\rangle}$, we infer that the triangle has two intersecting absolute lines $A, B$. The point $a=A \cap s^{\pi}$ is distinct from $A \cap B$. The plane generated by $A, B$ contains $s$. Thus the line as meets $B$; hence the absolute lines $A, B$, as are the sides of a triangle in $\mathrm{W}(F)$. This contradiction shows that $\mathrm{W}(F)^{\langle s\rangle}$ contains no triangle.

Let $x \in P^{\langle s\rangle}, L \in \mathscr{L}^{\langle s\rangle}$ with $x \notin L$. We want to show that $x$ is joined in $\mathrm{W}(F)^{\langle s\rangle}$ to some point on $L$. By the absence of triangles, $L \nsubseteq x^{\pi}$; hence $y=L \cap x^{\pi}$ has dimension 1 , and the assertion is clear if $y \in P^{\langle s\rangle}$. Now assume that $y \notin P^{\langle s\rangle}$; hence $y \in s^{\pi}$. Then $y \neq s$, since $x \notin s^{\pi}$.

If $L \in \mathscr{L}_{s} \backslash \mathscr{L}_{n}$, then $L=s y$ is absolute and belongs to $\mathscr{L}_{\pi}$, a contradiction. Thus $L \in \mathscr{L}_{\pi} \backslash \mathscr{L}_{s}$, and $L=L^{\pi}, x, s$ are contained in the projective plane $y^{\pi}$. Hence $x$ is joined to the intersection point $x s \cap L$; note that $x s \in \mathscr{L}^{\langle s\rangle}$, in view of $x \notin s^{\pi}$, and that $x s \cap L \in P^{\langle s\rangle}$, otherwise $x s \cap L=s \in L$, a contradiction.

We conclude that $\mathrm{W}(F)^{\langle s\rangle}$ is a generalized quadrangle. Every point is on $|F|+2$ lines, and every line carries $|F|$ points, thus $\mathrm{W}(F)^{\langle s\rangle}$ is thick if and only if $|F|>2$.

(1.3) REMARK. The generalized quadrangle $W\left(\mathbb{F}_{2}\right)^{\diamond}$ is the dual of the square grid of size $4 \times 4$, and $W\left(\mathbb{F}_{3}\right)^{\diamond}$ is isomorphic to the orthogonal quadrangle $\mathrm{Q}_{5}\left(\mathbb{F}_{2}\right)$.

These well-known facts are proved in Payne and Thas [18, 1.1, 5.3.2] by combinatorial methods; see Section 4 for a group-theoretic proof of $\mathrm{W}\left(\mathbb{F}_{3}\right)^{\ominus} \cong \mathrm{Q}_{5}\left(\mathbb{F}_{2}\right)$, and for further references. The generalized quadrangles 
$\mathrm{W}(F)^{\diamond}$ with $|F|>3$ behave differently, they are not Moufang quadrangles, see (2.5); cf. also Payne [17] and Payne and Thas [18, (6.2.4)] for the quadrangle $\mathrm{W}\left(\mathbb{F}_{4}\right)^{\diamond}$ of order $(3,5)$.

By construction, the group $\Gamma=\left(\mathrm{PSp}_{4} F\right)_{s}$ is a collineation group of both $\mathrm{W}(F)$ and $\mathrm{W}(F)^{\langle s\rangle}$. In fact, following [20], one can use $\Gamma$ in order to give another (group-theoretic) description of $\mathrm{W}(F)^{\langle s\rangle}$ :

(1.4) THEOREM. The slanted symplectic quadrangle $\mathrm{W}(F)^{\langle s\rangle}$ is represented by $\Gamma$ in the sense of [20]: the group $\Gamma$ is transitive on points, and the stabilizer $\Gamma_{p}$ of a point $p$ fixes the special line ps and acts transitively on the other (absolute) lines through $p$.

Proof. Clearly $p s$ is fixed by $\Gamma_{p}$. The transitivity assertions for $\Gamma$ and $\Gamma_{p}$ follow from Witt's lemma. Furthermore, $\Gamma$ preserves the special lines $\mathscr{L}_{s} \backslash \mathscr{L}_{\pi}$ and the absolute lines $\mathscr{L}_{\pi} \backslash \mathscr{L}_{s} ;$ hence the two orbits of $\Gamma_{p}$ on the lines through $p$ are not fused under the action of $\Gamma$. Thus $\mathrm{W}(F)^{\langle s\rangle}$ is represented by $\Gamma$.

(1.5) We give an explicit description of a representing triplet $\left(\Gamma, \Gamma_{p},\left\{\Gamma_{p s}, \Gamma_{L}\right\}\right)$ in the sense of [20]. Let the symplectic polarity $\pi$ be given by the alternating form

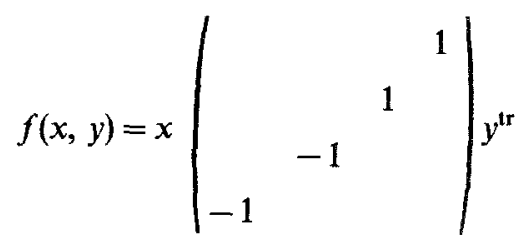

on $F^{4}$, and let $s=F(1,0,0,0)$. Then $\Gamma$ is induced by the group of all matrices

$$
\left(\begin{array}{lll}
x & & \\
w & A & \\
y & v & 1 / x
\end{array}\right)
$$

with $A \in \mathrm{SL}_{2} F, x \in F^{*}, y \in F, v \in F^{2}, w=A\left(\begin{array}{ll}1 & -1 \\ 1 & \end{array}\right) v^{\mathrm{tr}}$. The stabilizer $\Gamma_{p}$ of $p=F(0,0,0,1)$ is given by the conditions $v=w=0, y=0$, and $v=w=0$ defines the stabilizer $\Gamma_{p s}$ of the line ps. The stabilizer $\Gamma_{L}$ of the absolute line $L=\{0\} \times\{0\} \times F^{2}$ is induced by the matrices

$$
\left(\begin{array}{cccc}
x & & & \\
t & a & b & \\
& & 1 / a & \\
& & t & 1 / x
\end{array}\right)
$$


with $a, x \in F^{*}, b, t \in F$.

(1.6) COROLLARY. Each monomorphism $F \rightarrow F^{\prime}$ of fields yields a $\Gamma$-equivariant monomorphism $\mathrm{W}(F)^{\diamond} \rightarrow \mathrm{W}\left(F^{\prime}\right)^{\diamond}$ of generalized quadrangles.

This can be proved directly or by using $[21,3.5]$.

\section{Collineations}

The following result is well known, cf. Dieudonné [6, pp. 83/84]; we indicate a proof for the sake of completeness. Let $\mathrm{GSp}_{4} F$ denote the group of all symplectic similarities of $F^{4}$.

(2.1) THEOREM. Let $F, F^{\prime}$ be fields. Every isomorphism $\alpha: \mathrm{W}(F) \rightarrow \mathrm{W}\left(F^{\prime}\right)$ is induced by a unique collineation $\beta: \mathrm{PG}_{3} F \rightarrow \mathrm{PG}_{3} F^{\prime}$ satisfying $\pi \beta=\beta \pi^{\prime}$. In particular, $\mathrm{W}(F) \cong \mathrm{W}\left(F^{\prime}\right)$ if and only if $F \cong F^{\prime}$, and $\operatorname{Aut}(\mathrm{W}(F))$ $=\mathrm{PGSp}_{4} F \rtimes \operatorname{Aut}(F)$.

Proof. The non-absolute lines of $\mathrm{PG}_{3} F$ are the point sets $p^{\pi} \cap q^{\pi}$ with $p, q \in P, \quad p \notin q^{\pi}$. Thus $\alpha$ is (induced by) a unique collineation $\beta: \mathrm{PG}_{3} F \rightarrow \mathrm{PG}_{3} F^{\prime}$. Furthermore, $\quad \beta$ maps $\left\{\left(x, x^{\pi}\right) \mid x \in P\right\}$ onto $\left\{\left(x, x^{\pi^{\prime}}\right) \mid x \in P^{\prime}\right\}$, hence $\pi \beta=\beta \pi^{\prime}$. If $F=F^{\prime}$ and $\pi=\pi^{\prime}$, then $\beta$ is a semi-linear similarity.

(2.2) THEOREM. Let $F, F^{\prime}$ be fields containing at least five elements. Every isomorphism $\alpha: \mathrm{W}(F)^{\langle s\rangle} \rightarrow \mathrm{W}\left(F^{\prime}\right)^{\left\langle s^{\prime}\right\rangle}$ is induced by a unique collineation $\beta: \mathrm{PG}_{3} F \rightarrow \mathrm{PG}_{3} F^{\prime}$ satisfying $\pi \beta=\beta \pi^{\prime}$ and $s^{\beta}=s^{\prime}$ (hence also $s^{\pi \beta}=s^{\prime \pi^{\prime}}$ ).

Proof. First we describe the lines of the affine space $A G_{3} F$ in terms of $\mathrm{W}(F)^{\langle s\rangle}$. The star of a point $x$ in $\mathrm{W}(F)^{\langle s\rangle}$ is the set $x^{\pi} \cup x s$ (more precisely, the set of affine points in $x^{\pi}$ or on $\left.x s\right)$. For distinct points $x, y, z$ which are mutually not collinear in $\mathrm{W}(F)^{\langle s\rangle}$ we consider the set

$$
T_{x, y, z}=\left(x^{\pi} \cup x s\right) \cap\left(y^{\pi} \cup y s\right) \cap\left(z^{\pi} \cup z s\right) .
$$

Since $x s \cap y s=\varnothing$, etc., we have $T_{x, y, z}=\left(x^{\pi} \cap y^{\pi} \cap z^{\pi}\right) \cup S$, where $S$ contains at most three points. We claim that the affine lines missing in $\mathrm{W}(F)^{\langle s\rangle}$, i.e. the non-absolute affine lines not passing through $s$, are just the sets $T_{x, y, z}$ of cardinality at least 5 . Indeed, $\left|T_{x, y, z}\right| \geqslant 5$ implies that $x^{\pi} \cap y^{\pi} \cap z^{\pi}$ is a line $L$ of $\mathrm{PG}_{3} F$, hence $L^{\pi}=x y=x z=y z$. Thus $x^{\pi} \cap y^{\pi}=x^{\pi} \cap z^{\pi}=y^{\pi} \cap z^{\pi}=L$ contains $S$, and therefore $T_{x, y, z}=L$. Furthermore, given a non-absolute line $L$ not passing through $s$, we find distinct affine points $x, y, z$ on $L^{\pi} \notin \mathscr{L}_{\pi} \cup \mathscr{L}_{s}$, and then $T_{x, y, z}=L$ has cardinality $|F| \geqslant 5$.

This implies that $\alpha$ maps the lines of $\mathrm{AG}_{3} F$ onto the lines of $\mathrm{AG}_{3} F$, hence $\alpha$ is induced by a unique collineation $\beta: \mathrm{PG}_{3} F \rightarrow \mathrm{PG}_{3} F^{\prime}$ which maps the plane $s^{\pi}$ onto $s^{\prime \pi^{\prime}}$, cf. Tamaschke [22, 40.7, p. 229] (alternatively, disjoint lines of 
$\mathrm{AG}_{3} F$ are parallel precisely if they generate a proper affine subspace, hence $\alpha$ preserves parallelity and has therefore an extension $\beta$ as asserted, cf. Tamaschke $\left[22,40.3\right.$, p. 221]). The points of $s^{\pi} \backslash\{s\}$ are the points at infinity of the affine lines characterized above, hence $s^{\beta}=s^{\prime}$. Thus $\beta$ maps the special lines $\mathscr{L}_{s} \backslash \mathscr{L}_{\pi}$ onto $\mathscr{L}_{s^{\prime}}^{\prime} \backslash \mathscr{L}_{\pi^{\prime}}^{\prime}$, and the absolute lines $\mathscr{L}_{\pi} \backslash \mathscr{L}_{s}$ onto $\mathscr{L}_{\pi^{\prime}}^{\prime} \backslash \mathscr{L}_{s^{\prime}}^{\prime}$; furthermore, $\beta$ maps $\left\{L \in \mathscr{L} \mid s \in L \subseteq s^{\pi}\right\}=\mathscr{L}_{\pi} \cap \mathscr{L}_{s}$ onto $\mathscr{L}_{\pi^{\prime}}^{\prime} \cap \mathscr{L}_{s^{\prime}}^{\prime}$. This shows that $\beta$ is an isomorphism of $\mathrm{W}(F)$ onto $\mathrm{W}\left(F^{\prime}\right)$, hence $\pi \beta=\beta \pi^{\prime}$ by (2.1).

(2.3) COROLLARY. Let $F, F^{\prime}$ be fields. Then the slanted symplectic quadrangles $\mathrm{W}(F)^{\diamond}, \mathrm{W}\left(F^{\prime}\right)^{\diamond}$ are isomorphic if and only if $F$ and $F^{\prime}$ are isomorphic.

As a consequence of (2.2) and (1.4) we obtain

(2.4) COROLLARY. Let $F$ be a field containing at least five elements. Then

$$
\operatorname{Aut}\left(\mathrm{W}(F)^{\langle s\rangle}\right)=(\operatorname{Aut}(\mathrm{W}(F)))_{s}=\left(\mathrm{PGSp}_{4} F\right)_{s} \succ \operatorname{Aut}(F) ;
$$

this group is transitive on the points of $\mathrm{W}(F)^{\langle s\rangle}$, and it has two orbits on the lines, viz. $\mathscr{L}_{\pi} \backslash \mathscr{L}_{s}$ and $\mathscr{L}_{s} \backslash \mathscr{L}_{n}$.

The group Aut $\left(\mathbf{W}\left(\mathbb{F}_{4}\right)^{\circ}\right)$ has been determined by Payne [17]. The quadrangle $\mathrm{W}\left(\mathbb{F}_{4}\right)^{\diamond}$ is flag-homogeneous, but not a Moufang quadrangle, see Payne [17, V.4, V.5]. This shows that (2.2) and (2.4) are false for $F=\mathbb{F}_{4}$. Because Moufang quadrangles are (point- and) line-homogeneous, one obtains also

(2.5) COROLLARY. If $F$ is a field containing at least four elements, then $\mathrm{W}(F)^{\circ}$ is not a Moufang quadrangle.

\section{THE GROUPS OF PROJECTIVITIES}

Consider two disjoint lines $L, M$ in a generalized quadrangle $Q$. For each $x \in L$ there is a unique $y \in M$ such that $x$ and $y$ are collinear. The mapping $[L, M]: L \rightarrow M$ defined in this way is called the perspectivity from $L$ to $M$. Given lines $L_{0}, L_{1}, \ldots, L_{k}$ with $L_{i} \cap L_{i+1}=\varnothing$, for $0 \leqslant i<k$, the product $\left[L_{0}, L_{1}, \ldots, L_{k}\right]=\left[L_{0}, L_{1}\right]\left[L_{1}, L_{2}\right] \ldots\left[L_{k-1}, L_{k}\right]$ is called a projectivity from $L_{0}$ to $L_{k}$. The projectivities of a fixed line $L$ back to itself form a group $\Pi(L)$, the group of projectivities of $L$. It has a normal subgroup $\Pi^{+}(L)$ of index at most 2 consisting of those projectivities which can be written as a product of an even number of perspectivities. $\Pi^{+}(L)$ is called the group of even projectivities.

In a thick quadrangle, there is a projectivity $\sigma$ from any line $L$ to any other line $M$; the equations $\Pi(L)=\sigma^{-1} \Pi(M) \sigma$ and $\Pi^{+}(L)=\sigma^{-1} \Pi^{+}(M) \sigma$ show that the groups of projectivities of $L$ and $M$ are isomorphic as permutation 
groups. Thus these permutation groups are invariants of the generalized quadrangle $Q$.

(3.1) PROPOSITION (Knarr [12, 1.2]). For every thick generalized quadrangle, the group $\Pi^{+}$of even projectivities is doubly transitive.

Knarr [12] has determined the groups of projectivities of all finite Moufang polygons. His arguments for symplectic quadrangles $\mathrm{W}(F)$ and their duals $\mathrm{W}(F)^{\text {dual }} \cong \mathrm{Q}_{4}(F)$ hold also for infinite fields $F$, hence one has the following result.

(3.2) THEOREM (Knarr). Let $F$ be a field. For the symplectic quadrangle $\mathrm{W}(F)$, we have $\Pi=\Pi^{+}=\mathrm{PGL}_{2} F$ in its natural action on the projective line $F \cup\{\infty\}$. For the dual quadrangle $\mathrm{W}(F)^{\mathrm{dual}} \cong \mathrm{Q}_{4}(F)$, we have $\Pi=\Pi^{+}=\mathrm{PSL}_{2} F$ in its natural action on the projective line $F \cup\{\infty\}$.

In this section we determine the groups of projectivities of the slanted symplectic quadrangles $\mathrm{W}(F)^{\diamond}$ and their duals. We make the general assumption that $|F|>2$, so $W(F)^{\diamond}$ is thick by (1.2).

For the rest of this section let $\Pi$ and $\Pi^{+}$denote the group of projectivities and the group of even projectivities of $\mathrm{W}(F)^{\diamond}$, respectively. Let $\Pi_{*}$ and $\Pi_{*}^{+}$be the group of projectivities and the group of even projectivities of $\mathrm{W}(F)^{\diamond \text { dual }}$, respectively.

For a set $X$, denote by $\operatorname{Sym}(X)$ the symmetric group on $X$, and by $\operatorname{Sym}_{\text {fin }}(X)$ the finitary symmetric group on $X$, i.e. the group of all permutations moving only finitely many elements of $X$. Furthermore, $\operatorname{Alt}(X)$ denotes the group of all finitary even permutations of $X$.

(3.3) THEOREM. Let $F$ be a field with $|F|>2$. For the slanted symplectic quadrangle $\mathrm{W}(F)^{\diamond}$ we have

$$
\Pi=\Pi^{+}=\operatorname{Sym}(F),
$$

if $F$ is finite. For infinite fields $F$, the group

$$
\Pi=\Pi^{+}=\left(\operatorname{Sym}_{\mathrm{fin}}(F \cup\{\infty\}) \succ \mathrm{PGL}_{2} F\right)_{\infty}
$$

is the stabilizer of $\infty$ in the product of the finitary symmetric group and $\mathrm{PGL}_{2} F$ in their natural actions on $F \cup\{\infty\}$.

In particular, one has $\operatorname{Sym}_{\mathrm{fin}}(F) \leqslant \Pi=\Pi^{+}$.

(3.4) THEOREM. Let $F$ be a field with $|F|>2$. For the dual $\mathrm{W}(F)^{\diamond \mathrm{dual}}$ of the slanted symplectic quadrangle, we have

$$
\left.\Pi_{*}^{+}=\operatorname{Alt}\left(F \cup\left\{\infty, \infty^{\prime}\right\}\right)\right) \text { and } \Pi_{*}=\operatorname{Sym}\left(F \cup\left\{\infty, \infty^{\prime}\right\}\right) \text {, }
$$


if $F$ is finite. For infinite fields $F$, we have

$$
\Pi_{*}^{+}=\operatorname{Alt}\left(F \cup\left\{\infty, \infty^{\prime}\right\}\right) \rtimes \mathrm{PSL}_{2} F
$$

and

$$
\Pi_{*}=\operatorname{Sym}_{\text {fin }}(F \cup\{\infty, \infty\}) \succ \operatorname{PSL}_{2} F,
$$

where $\operatorname{PSL}_{2} F$ acts naturally on $F \cup\{\infty\}$ and fixes $\infty$.

In particular, one has $\operatorname{Alt}(F \cup\{\infty, \infty\}) \leqslant \Pi_{*}^{+}<\Pi_{*}$.

These results show that rather large subgroups of the groups of projectivities of the classical symplectic quadrangle survive as subgroups of $\Pi$ and $\Pi_{*}$; this is due to the fact that the automorphism group remains rather large, cf. Knarr [12,1.3]. Furthermore, these results support a conjecture of Knarr [12] which says that in every finite non-classical generalized quadrangle, the group of even projectivities should contain the alternating group.

Now we prepare the proofs of $3.3,3.4$.

(3.5) LEMMA. $\Pi=\Pi^{+}$.

Proof. Take distinct special lines $L_{0}, L_{1}, L_{2} \in \mathscr{L}_{s} \backslash \mathscr{L}_{n}$ in some affine subplane of $\mathrm{AG}_{3} F$. Any line meeting $L_{0}$ and $L_{1}$ also meets $L_{2}$, hence $\left[L_{0}, L_{1}, L_{2}, L_{0}\right]=1$, which implies $\Pi=\Pi^{+}$.

(3.6) LEMMA. AGL $F \leqslant \Pi$.

Proof. We introduce coordinates in $\mathrm{W}(F)^{\diamond}=\mathrm{W}(F)^{\langle s\rangle}$ as in (1.5). Thus $s=F(1,0,0,0)$ and $s^{\pi}=F^{3} \times\{0\}$. Let $(a, b, c)=F(a, b, c, 1) \in P^{\langle s\rangle}=P \backslash s^{\pi}$ denote an affine point. Then the absolute lines through $(a, b, c)$ are given as sets $(a, b, c)+F(b \gamma-\beta c, \beta, \gamma)$, where $(\beta, \gamma) \in F^{2} \backslash\{(0,0)\}$. The unique special line through $(a, b, c)$ is $(a, b, c)+F(1,0,0)$.

The special lines $X=(0,0,1)+F(1,0,0), \quad M=(0,0, m)+F(1,0,0)$, $N=F(1,0,0), B=(0, b, 0)+F(1,0,0)$ with $m \notin\{0,1\}$ and $b \neq 0$ are mutually disjoint. Furthermore, the absolute line $A=F(0,1,0)$ is disjoint from $X$ and $M$.

Let $x \in F$. We compute $(x, 0,1)^{[X, A]}=(0, x, 0),(0, x, 0)^{[A, M]}=(m x, 0, m)$, and $(m x, 0, m)^{[M, x]}=(m x, 0,1)$. On the other hand, $(x, 0,1)^{[X, B]}=(x+b, b, 0)$, $(x+b, b, 0)^{[B, N]}=(x+b, 0,0)$, and $(x+b, 0,0)^{[N, X]}=(x+b, 0,1)$.

These transformations generate $\mathrm{AGL}_{1} F$.

(3.7) LEMMA. $\operatorname{Sym}_{\text {fin }}(F) \leqslant \Pi$.

Proof. We use the same coordinatization as in the previous lemma.

The absolute lines $L=F(0,0,1)$ and $M=(1,0,0)+F(0,1,1)$ are disjoint, and $(0,0, x) \in L$ is joined to $\left(1,-x^{-1},-x^{-1}\right) \in M$ by an absolute line for 
$x \neq 0$, whereas $(0,0,0)$ and $(1,0,0)$ are joined by a special line. This means that $(0,0, x)^{[L, M]}=\left(1, x^{\prime}, x^{\prime}\right)$ with $x^{\prime}=-x^{-1}$ for $x \neq 0$, and $0^{\prime}=0$. Similarly, with the special line $N=(0,-1,0)+F(1,0,0)$ we have $(1, x, x)^{[M, N, L]}=$ $(x+1,-1,0)^{[N, L]}=(0,0, x+1)$ for all $x \in F$. Hence $\sigma=[L, M, N, L]$ satisfies $(0,0, x)^{\sigma}=\left(0,0,1+x^{\prime}\right)$ for all $x \in F$. The permutation $x \mapsto 1+x^{\prime}: F \rightarrow F$ exchanges 0 and 1 , and has cycles of length 3 on $F \backslash\{0,1\}$. Thus $\sigma^{3}$ is a transposition, and the assertion follows with (3.1).

(3.8) LEMMA. $\Pi \leqslant\left(\operatorname{Sym}_{\text {fin }}(F \cup\{\infty\})>\triangle \mathrm{PGL}_{2} F\right)_{\infty}$.

Proof. It is sufficient to show that any perspectivity $[L, M]$ agrees with some projective collineation of the surrounding projective space $\mathrm{PG}_{3} F$ on all but finitely many points.

Let $L$ be an absolute line, i.e. $L \in \mathscr{L}_{\pi} \backslash \mathscr{L}_{s}$. Let $M$ be another line, absolute or special, which is disjoint from $L$. Then $\left|L \cap M^{\pi}\right| \leqslant 1$. If $x \in L \backslash M^{\pi}$ is an affine point, then $M \nsubseteq x^{\pi}$, hence $x^{\prime}=x^{\pi} \cap M$ is a point in $x^{\pi}$. Furthermore, $x x^{\prime} \in \mathscr{L}_{n} \backslash \mathscr{L}_{s}$, in view of $s \notin x^{\pi}$, hence $x^{[L, M]}=x^{\prime}=x^{\pi} \cap M$. The mapping $x \mapsto x^{\pi} \cap M$ is induced by a projective collineation of $\mathrm{PG}_{3} F$.

It remains to consider two special lines $L, M \in \mathscr{L}_{s} \backslash \mathscr{L}_{n}$. They span a projective plane $p^{\pi}$ in $P G_{3} F$. Let $x \in L$ and $x^{\prime}=x^{[L, M]}$. Then $x x^{\prime}=\left(x x^{\prime}\right)^{\pi}$ contains $\left(p^{\pi}\right)^{\pi}=p$, hence $x^{[L, M]}=x^{\prime}=x p \cap M$. Thus $[L, M]$ is a perspectivity of the projective plane $p^{\pi} \cong \mathrm{PG}_{2} F$.

PROOF OF THEOREM (3.3). The assertion follows from (3.5) and (3.7) in the finite case. Now let $F$ be infinite. The group $\Pi$ contains $\mathrm{AGL}_{1} F=\left(\mathrm{PGL}_{2} F\right)_{\infty}$ and $\operatorname{Sym}_{\mathrm{fin}}(F)=\operatorname{Sym}_{\mathrm{fin}}(F \cup\{\infty\})_{\infty}$ by (3.6) and (3.7), respectively.

Take $\sigma \in \Pi$ as in the proof of (3.7) and assume that $\sigma \in \operatorname{Sym}_{\text {fin }} F \rtimes \mathrm{AGL}_{1} F$. Then there exists $m \in F \backslash\{0\}$ such that $x^{\sigma}-y^{\sigma}=(x-y) m$, i.e. $m x y=1$, for all but finitely many $x, y \in F$, a contradiction.

In view of (3.8), the assertion follows from the following lemma.

(3.9) LEMMA. Let $X$ be infinite and let $S=\operatorname{Sym}_{\text {fin }}(X)$. If $P$ is a primitive subgroup of $\operatorname{Sym}(X)$ with $P \cap S=1$, then $S_{x} P_{x}$ is a maximal subgroup of $(S P)_{x}$ for every $x \in X$.

Proof. Let $Y=2^{X} / \sim$ be the quotient of the power set $2^{X}$, where $A \sim B$ means that the symmetric difference $A+B=(A \cup B) \backslash(A \cap B)$ is finite (in other words, $Y$ is the quotient of the group $\left(2^{X},+\right)$ modulo the subgroup consisting of all finite subsets of $X$ ). Then $S$ is the kernel of the natural action $\varphi$ of $\operatorname{Sym}(X)$ on $Y$. Let $x \in X$. We claim that $\varphi(P)=\varphi\left((S P)_{x}\right)$; indeed, $\varphi(p)=\varphi(s p)$ for $p \in P, s \in S$, and we can choose $s$ such that $x^{s p}=x$.

By primitivity, $P_{x}$ is maximal in $P$, and $\varphi\left(P_{x}\right)=\varphi(P)$ implies $P \subseteq S P_{x}$ and $P=S P_{x} \cap P=(S \cap P) P_{x}=P_{x}$, a contradiction. Hence $\varphi\left(P_{x}\right)$ is maximal in 
$\varphi(P)=\varphi\left((S P)_{x}\right)$. Since $S_{x}$ is the kernel of $\varphi$ restricted to $(S P)_{x}$, we infer that $S_{x} P_{x}$ is maximal in $(S P)_{x}$.

Now we examine the dual groups $\Pi_{*}$ and $\Pi_{*}^{+}$. The points and lines of the dual quadrangle will be viewed as lines and points, respectively, of the slanted symplectic quadrangle.

(3.10) LEMMA. $\operatorname{Sym}_{\text {fin }}(F \cup\{\infty, \infty\}) \leqslant \Pi_{*}$.

Proof. Let $p, q, r \in P^{\langle s\rangle}$ be distinct points which lie on a non-absolute line $L \in \mathscr{L} \backslash \mathscr{L}_{s}$. Then the projectivity $[p, q, r, p]$ of $\mathrm{W}(F)^{\text {dual }}$ is the identity (dualize the proof of (3.5)).

We claim that the projectivity $\sigma=[p, q, r, p]$ of $\mathrm{W}(F)^{\langle s\rangle \text { dual }}$ is a transposition; then the assertion follows with (3.1). Indeed, using the point $t=L^{\pi} \cap\langle L, s\rangle$, we compute

$$
(p s)^{\sigma}=(q t)^{[q, r, p]}=(r s)^{[r, p]}=p t,
$$

because the lines $p s, q t$ intersect in a point of $\langle L, s\rangle \backslash s^{\pi}$, as well as the lines $q t$, $r s$ and $r s, p t$. If $X \neq p s, p t$ is a line through $p$, then $X^{\sigma}=X$ as in the classical quadrangle $\mathrm{W}(F)^{\text {dual }}$. Hence $\sigma$ is the transposition which exchanges $p s$ and $p t$.

(3.11) COROLLARY. Alt $\left(F \cup\left\{\infty, \infty^{\prime}\right\}\right) \leqslant \Pi_{*}^{+}$.

PROOF OF THEOREM (3.4). Take two points $p, q \in P^{\langle s\rangle}$ which are not joined. Then $p, q, s$ span a projective plane in $\mathrm{PG}_{3} F$, and $t=\langle p, q, s\rangle \cap p^{\pi} \cap q^{\pi}$ is a point. The perspectivity $[p, q]$ of $\mathrm{W}(F)^{\langle s\rangle}$ dual agrees on all lines $X \neq p s, p t$ through $p$ with the corresponding perspectivity $\alpha$ of the classical quadrangle $\mathrm{W}(F)^{\mathrm{dual}}$, and $p x^{[p, q]}=q s$ and $p s^{[p, q]}=q x$. Thus $[p, q]$ differs by a transposition from $\alpha$, if we extend $\alpha$ by defining $(p s)^{\alpha}=p s$. As a consequence, $\Pi_{*}^{+} \leqslant \operatorname{Alt}(F \cup\{\infty, \infty \prime)) \mathrm{PSL}_{2} F$ and $\Pi_{*} \leqslant \operatorname{Sym}_{\mathrm{fin}}\left(F \cup\{\infty, \infty \prime) \mathrm{PSL}_{2} F\right.$. Since $\mathrm{PSL}_{2} F$ is contained in the alternating group for finite fields $F$ with $|F|>2$, the assertions for the finite case follow.

Let $F$ be infinite. The symplectic group $\mathrm{PSp}_{4} F$ is generated by the symplectic transvections with centers in $P \backslash s^{\pi}$. On pencils of absolute lines, this group $\mathrm{PSp}_{4} F$, and $\left(\mathrm{PSp}_{4} F\right)_{s}$ as well, induce the group $\mathrm{PSL}_{2} F$ of even projectivities of $\mathrm{W}(F)^{\text {dual }}$, see (3.2) and Knarr $[12,1.3,2.1]$. Consider $\sigma \in\left(\mathrm{PSp}_{4} F\right)_{s}$ with center $c \in P \backslash s^{\pi}$. Let $p \in P \backslash s^{\pi}$. We find $x \in P \backslash\left(s^{\pi} \cup p^{\pi} \cup c^{\pi} \cup p s \cup c s\right)$, as $F$ is infinite. The proof in Knarr [12,1.3] shows that the restriction of $\sigma$ to the pencil of absolute lines through $p$ coincides with the even projectivity $\left[p, x, c, x^{\sigma}, p^{\sigma}\right]$ of $\mathrm{W}(F)^{\mathrm{dual}}$. This even projectivity differs from the even projectivity $\left[p, x, c, x^{\sigma}, p^{\sigma}\right]$ of $\mathrm{W}(F)^{\diamond \text { dual }}$ by an element of $\operatorname{Alt}(F \cup\{\infty, \infty)\}$. From (3.11) we conclude that $\mathrm{PSL}_{2} F \leqslant \Pi_{*}^{+}$.

In order to formulate an (anti)topological application, we recall that a 
topological quadrangle is a generalized quadrangle $Q=(P, \mathscr{L}, \epsilon)$ with nondiscrete Hausdorff topologies on $P$ and on $\mathscr{L}$ such that the geometric operations in $Q$ are continuous. This definition implies that all perspectivities and all projectivities are homeomorphisms, cf. [10,2.2].

(3.12) THEOREM. There are no (non-discrete Hausdorff) topologies on $P^{\diamond}$ and $\mathscr{L}^{\diamond}$ which render $\mathrm{W}(F)^{\diamond}$ a topological quadrangle.

Proof. Assume the contrary. Then each line $L \in \mathscr{L}^{\diamond}$ is a non-discrete Hausdorff space, cf. [10,2.8], hence every cofinite subset of $L$ is dense in $L$. By (3.7) there are non-trivial homeomorphisms of $L$ onto itself which fix all but finitely many points of $L$, a contradiction.

According to Szambien [23, Prop. 1], there exist projective planes (i.e. generalized triangles) which cannot be made into (non-discrete Hausdorff) topological projective planes; the construction of these planes is more difficult and less direct.

\section{APPENDIX}

In this appendix we prove by a group-theoretic argument that $\mathrm{W}\left(\mathbb{F}_{3}\right)^{\diamond}$ is isomorphic to the orthogonal quadrangle $\mathrm{Q}_{5}\left(\mathbb{F}_{2}\right)$, cf. Payne and Thas $[18$, 5.3.2]. This configuration of 27 points and 45 lines has a venerable history: it is dual to the 27 lines (and their intersection points) on a general cubic surface, and its collineation group is isomorphic to the Weyl group $\mathrm{E}_{6}$, see Hartshorne [11, pp. 401-405], Mumford [14, §8D], Coxeter [3], Miller $e$ t al. [13, Ch. XIX]; cf. also Payne and Thas [18, 6.1], Freudenthal [8] and Frame [7].

The standard hermitian form $\Sigma_{i=1}^{3} x_{i} \bar{x}_{i}$ on $\left(\mathbb{F}_{4}\right)^{3}$ can be considered as a nondegenerate quadratic form on $\left(\mathbb{F}_{2}\right)^{6}$ of Witt index 2 (it is also a cubic form over $\left.\mathbb{F}_{4}\right)$; a non-zero vector $\left(x_{1}, x_{2}, x_{3}\right) \in\left(\mathbb{F}_{4}\right)^{3}$ is singular if and only if precisely one coordinate $x_{i}$ is 0 . The quadrangle $\mathrm{Q}_{5}\left(\mathbb{F}_{2}\right)$ consists of the totally isotropic subspaces of this quadratic form on $\left(\mathbb{F}_{2}\right)^{6}$.

The group $\Delta=\mathrm{SU}_{3} \mathbb{F}_{4}$ of order $2^{3} \cdot 3^{3}$ acts faithfully as a collineation group of $\mathrm{Q}_{5}\left(\mathbb{F}_{2}\right)$; the Sylow 2-subgroups of $\Delta$ are quaternion groups.

(4.1) LEMMA. The quadrangle $Q_{5}\left(\mathbb{F}_{2}\right)$ is represented by $\Delta$ in the sense of [20]: the group $\Delta$ is transitive on the points of $\mathrm{Q}_{5}\left(\mathbb{F}_{2}\right)$, and it has two orbits on the lines. The stabilizer $\Delta_{p}$ of a point $p$ is a Sylow 2-subgroup of $\Delta$, and $\Delta_{p}$ fixes a unique line $L$ through $p$ and is transitive on the other lines through $p$. Furthermore $\Delta_{L}=\Delta_{p} \times Z$ is the direct product of $\Delta_{p}$ and the center $Z$ of $\Delta$, and if $M$ is one of the other lines through $p$, then $\Delta_{M}$ is isomorphic to the symmetric group $S_{3}$ of degree 3 , and $\Delta_{M} \cap \Delta_{p}$ is the center of $\Delta_{p}$. 
Proof. By Witt's lemma, and by adjusting the determinant, $\Delta$ is transitive on the 27 points and on the pairs $(x, y)$ of $\mathbb{F}_{4}$-independent vectors $x, y$ such that $x, y, x+y$ are isotropic. Thus the stabilizers of points are precisely the Sylow 2-subgroups of $\Delta$. The stabilizer of a point $p=\mathbb{F}_{2} x$ fixes the line $L=\mathbb{F}_{4} x$, and $\Delta_{L}=\Delta_{x} Z=\Delta_{p} Z$, as $Z=\mathbb{F}_{4}^{*}$ is transitive on $L \backslash\{0\}$. We have $M=\{x, y, x+y, 0\}$ with $x, y$ as above, hence $\Delta_{M}$ induces $S_{3}$ on $M$, and $\Delta_{x, y}=1$, as $x, y$ are $\mathbb{F}_{4}$-independent. Finally $\Delta_{M} \cap \Delta_{p}$ has order 2 , hence it coincides with the center of $\Delta_{p}$.

Now we consider groups over $\mathbb{F}_{3}$. Let $\Sigma$ be the (normal) Sylow 2-subgroup of $\mathrm{SL}_{2} \mathbb{F}_{3}$. We claim that the group

$\Gamma_{0}=\left\{\left(\begin{array}{ccc}1 & \\ w & A \\ z & v & 1\end{array}\right) \mid A \in \Sigma, v, w \in \mathbb{F}_{3}^{2}, z \in \mathbb{F}_{3}, w=A\left(\begin{array}{rr} & 1 \\ -1 & \end{array}\right) v^{\mathrm{ir}}\right\}$

is isomorphic to $\Delta$. Indeed, both $\Gamma_{0}$ and $\Delta$ have normal Sylow 3-subgroups $E$ which are isomorphic (they are extra-special groups of order 27 and exponent 3, cf. Gorenstein $[9,5.5 .1$, p. 203]). Furthermore the Sylow 2-subgroups are quaternion groups $\Sigma$ of order 8 which act faithfully on the center factor groups $E / Z(E)$. We infer that all semidirect products $E \rtimes \Sigma$ with these properties are isomorphic, because $A u t(E / Z(E)) \cong G L_{2} \mathbb{F}_{3}$ contains only one copy of $\Sigma$ (note that all six elements of order 4 in $\mathrm{GL}_{2} \mathbb{F}_{3}$ are contained in $\Sigma$ ). Thus $\Gamma_{0} \cong \Delta$.

It is easy to verify that $\Gamma_{0}$ represents $W\left(\mathbb{F}_{3}\right)^{\diamond}$, and that all assertions in (4.1) hold for $\left(\Gamma_{0}, W\left(\mathbb{F}_{3}\right)^{\ominus}\right)$ instead of $\left(\Delta, Q_{5}\left(\mathbb{F}_{2}\right)\right)$. For each Sylow 2-subgroup $\Sigma$ of $\Gamma_{0} \cong \Delta$, the copies of $S_{3}$ containing the center of $\Sigma$ are conjugate under $\Sigma$. Therefore every isomorphism $\Delta \rightarrow \Gamma_{0}$ maps the representing triplet $\left(\Delta, \Delta_{p},\left\{\Delta_{L}, \Delta_{M}\right\}\right)$ for $\mathrm{Q}_{5}\left(\mathbb{F}_{2}\right)$ to a representing triplet for $\mathrm{W}\left(\mathbb{F}_{3}\right)^{\ominus}$, and from [20], (2), we obtain an isomorphism $\varphi: \mathrm{Q}_{5}\left(\mathbb{F}_{2}\right) \rightarrow \mathrm{W}\left(\mathbb{F}_{3}\right)^{\diamond}$.

Note that $\Gamma_{0}$ acts faithfully on $W\left(\mathbb{F}_{3}\right)^{\diamond}$, inducing a subgroup of index 6 in the group $\Gamma$ defined in Section 1 (with $F=\mathbb{F}_{3}$ ). We remark that the group $\Gamma U_{3} \mathbb{F}_{4}=\mathrm{U}_{3} \mathbb{F}_{4} \rtimes \operatorname{Aut}\left(\mathbb{F}_{4}\right)$ contains $\Delta=\mathrm{SU}_{3} \mathbb{F}_{4}$ as a subgroup of index 6 and satisfies the transitivity assumptions in (4.1), hence $\Gamma=\varphi^{-1}\left(\Gamma U_{3} \mathbb{F}_{4}\right) \varphi$ is isomorphic to $\Gamma U_{3} \mathbb{F}_{4}$. However, it seemed easier to work with the smaller groups $\Delta$ and $\Gamma_{0}$.

\section{REFERENCES}

1. Bichara, A., 'Characterization of the generalized quadrangles embedded in $A_{3, q}$, Riv. Mat. Univ. Parma (4) 4 (1978), 277-290.

2. Bichara, A., Mazzocca, F. and Somma, C., 'On the classification of generalized quadrangles in finite affine spaces $\mathrm{AG}\left(3,2^{h}\right)^{\prime}$, Boll. Un. Mat. Ital. (5) 17-B (1980), 298-307. 
3. Coxeter, H. S. M., 'The evolution of Coxeter-Dynkin diagrams', Nieuw Arch. Wisk. (4) 9 (1991), 233-248.

4. De Soete, $M$. and Thas, J. A., 'Characterizations of the generalized quadrangles $\mathrm{T}_{2}^{*}(O)$ and $\mathrm{T}_{2}(\mathrm{O})$ ', Ars Combin. 22 (1986), 171-186.

5. De Soete, M. and Thas, J. A., ' $R$-Regularity and characterizations of the generalized quadrangles $\mathrm{P}(\mathrm{W}(\mathrm{s}),(\infty))$ ), Proc. Bari 1984 (eds A. Barlotti et al.), Ann. Discrete Math. 30 (1986), 171-184.

6. Dieudonné, J., La géométrie des groupes classiques, 3rd edn, Springer, Berlin, 1971.

7. Frame, J. S., 'A symmetric representation of the twenty-seven lines on a cubic surface by lines in a finite geometry', Bull. Amer. Math. Soc. 44 (1938), 658-661.

8. Freudenthal, H., 'Une étude de quelques quadrangles généralisés', Ann. Mat. Pura Appl. (4) 102 (1975), 109-133.

9. Gorenstein, D., Finite Groups, Harper \& Row, New York, 1968.

10. Grundhöfer, T. and Knarr, N., 'Topology in generalized quadrangles', Topology Appl. 34 (1990), 139-152.

11. Hartshorne, R., Algebraic Geometry, Springer, New York, 1977.

12. Knarr, N., 'Projectivities in generalized polygons', Ars Combin. 25B (1988), 265-275.

13. Miller, G. A., Blichfeld, H. F. and Dickson, L. E., Theory and Applications of Finite Groups, Stechert, New York, 1938.

14. Mumford, D., Algebraic Geometry I, Complex Projective Varieties, Springer, Berlin, 1976.

15. Payne, S. E., 'Nonisomorphic generalized quadrangles', J. Algebra 18 (1971), 201-212.

16. Payne, S. E., 'The equivalence of certain generalized quadrangles', J. Combin. Theory Ser. A 10 (1971), 284-289.

17. Payne, S. E., 'The generalized quadrangle with $(s, t)=(3,5)$ ', Proc. Boca Raton 1990, Congr. Numerantium 77 (1990), 5-29.

18. Payne, S. E. and Thas, J. A., Finite Generalized Quadrangles, Pitman, London, 1984.

19. Somma, C., 'Generalized quadrangles with parallelism', Proc. Trento 1980 (ed. A. Barlotti), Annals Discrete Math. 14 (1982), 265-282.

20. Stroppel, M., 'Reconstruction of incidence structures from groups of automorphisms', Arch. Math. (Basel) 58 (1992), 621-624.

21. Stroppel, M., 'A categorical glimpse at the reconstruction of geometries', Geom. Dedicata (to appear).

22. Tamaschke, O., Projektive Geometrie, II, Bibliogr. Inst., Mannheim, 1972.

23. Szambien, H., 'Minimal topological projective planes', J. Geometry 35 (1989), 177-185.

Authors' addresses:

T. Grundhöfer and M. Joswig,

Mathematisches Institut,

Universität Tübingen,

Auf der Morgenstelle 10,

D-7400 Tübingen,

Germany.
M. Stroppel,

Fachbereich Mathematik,

Technische Hochschule Darmstadt,

Schlossgartenstr. 7,

D-6100 Darmstadt,

Germany.

(Received, November 4, 1992; revised version, December 7, 1992) 